\title{
THE EXISTENCE OF NONMEASURABLE SETS FOR INVARIANT MEASURES
}

\author{
MARCIN PENCONEK AND PIOTR ZAKRZEWSKI
}

(Communicated by Andreas Blass)

\begin{abstract}
We prove that if $G$ is a locally compact Polish group acting in a reasonable way on a set $X$, then for every countably additive, $\sigma$-finite, $G$ invariant measure on $X$ there exist nonmeasurable sets. In particular, the latter is true when $X$ is a compact, metric, metrically homogeneous space, and $G$ is the group of its isometries.
\end{abstract}

\section{INTRODUCTION AND PRELIMINARIES}

By a measure on a set $X$ we mean a countably additive, nonzero function defined on a $\sigma$-algebra of subsets of $X$ and assuming values in $[0,+\infty]$. A measure is nonatomic if it vanishes on all singletons; it is $\sigma$-finite if $X$ is a countable union of sets of finite measure.

A measure on $X$ is universal if it is defined on the $\sigma$-algebra $\mathscr{P}(X)$ of all subsets of $X$; i.e., nonmeasurable sets do not exist.

Suppose that a group $G$ acts on a set $X$, by which we mean that there is a function $\langle g, x\rangle \rightarrow g x$ of $G \times X$ into $X$ such that

(i) for each $g \in G, x \rightarrow g x$ is a permutation of $X$,

(ii) for all $x \in X$ and $g_{1}, g_{2} \in G, g_{1}\left(g_{2} x\right)=\left(g_{1} \cdot g_{2}\right) x$.

For any set $A \subseteq X$ and any $g \in G$ we write $g A=\{g x: x \in A\}$.

A measure $m: \mathscr{A} \rightarrow[0,+\infty]$ on $X$ is $G$-invariant if $g A \in \mathscr{A}$ and $m(A)=$ $m(g A)$ whenever $A \in \mathscr{A}$ and $g \in G$.

For any $x \in X, G x=\{g x: g \in G\}$ and $G_{x}=\{g \in G: g x=x\}$ are the $G$-orbit and the stabilizer of $x$, respectively.

Although the existence of a set carrying a $\sigma$-finite, nonatomic, universal measure cannot be proved in ZFC (see [1, Chapter 6]), direct arguments employing invariance properties of measures to show the existence of nonmeasurable sets within ZFC have always been of considerable interest (see, e.g., [2-4]). The Vitali construction of a Lebesgue nonmeasurable set is the prototype of such results.

Received by the editors July 29, 1991 and, in revised form, September 29, 1992.

1991 Mathematics Subject Classification. Primary 03E15, 28A05; Secondary 28C10, $03 E 05$.

Key words and phrases. Action by a locally compact Polish group, invariant $\sigma$-finite measure, nonmeasurable set.

The main result of this paper was obtained while the second author was visiting the Technische Universität Berlin as a research fellow of the Alexander von Humboldt Foundation. 
This paper belongs to the same line of work. Its principal aim is to show that for simple enough actions by locally compact Polish groups the invariance of a $\sigma$-finite measure together with the uncountability of all orbits implies the existence of nonmeasurable sets (Theorem 2.1). This and two related results (Theorems 3.3 and 3.4) provide a partial answer to the following problem which remains still open.

Question. Let $G$ be a Polish group acting in a nice way on a space $X$ with each $G$-orbit uncountable. If $m$ is a $\sigma$-finite $G$-invariant measure on $X$, does $m$ have a nonmeasurable set?

\section{MAIN RESUlt}

Our main result is the following.

2.1. Theorem. Suppose that $G$ is a locally compact Polish group and let $\lambda: \mathscr{B}$ $\rightarrow[0,+\infty]$ be the completion of a left-invariant Haar measure on $G$. Suppose further that $G$ acts on a set $X$ in such a way that the stabilizers of all points of $X$ are $\lambda$-measurable and all $G$-orbits are uncountable. Then for every $G$-invariant $\sigma$-finite measure on $X$ there exist nonmeasurable sets.

Proof. Let $m$ be a $G$-invariant $\sigma$-finite measure on $X$ and suppose, towards a contradiction, that $m$ is universal. Notice that the $\sigma$-finiteness of $m$ and the uncountability of all $G$-orbits implies that the measure $m$ is nonatomic. We have

Claim. There exists a countable subgroup $H$ of $G$ such that, for every $g \in G, m(\{x \in X: g x \in H x\})>0$.

In order to prove the claim, suppose that such a subgroup does not exist. Using this assumption, define by induction on $\alpha<\omega_{1}$ an increasing sequence $\left\langle H_{\alpha}: \alpha<\omega_{1}\right\rangle$ of countable subgroups of $G$ and a sequence $\left\langle g_{\alpha}: \alpha<\omega_{1}\right\rangle$ of elements of $G$ keeping the conditions $m\left(\left\{x \in X: g_{\alpha} x \in H_{\alpha} x\right\}\right)=0$ and $g_{\alpha} \in$ $H_{\alpha+1}$ satisfied for every $\alpha<\omega_{1}$. Finally, let $A=\bigcup_{\alpha<\omega_{1}}\left\{x \in X: g_{\alpha} x \in H_{\alpha} x\right\}$ and $G^{\prime}=\bigcup_{\alpha<\omega_{1}} H_{\alpha}$. Notice that

$$
m(A)=0,
$$

since $m$ being a $\sigma$-finite, $\sigma$-additive, nonatomic universal measure, is $\omega_{2}$ additive; i.e., every union of $\omega_{1}$ many sets of measure zero has measure zero (see [1, p. 176]).

Set $B=X \backslash A$. The subgroup $G^{\prime}$ has cardinality $\omega_{1}$ and the $G^{\prime}$-orbit of every element of $B$ is uncountable, and so also of power $\omega_{1}$. Hence the set $G^{\prime} B=\bigcup_{g \in G} g B$ can be partitioned into $\omega_{1}$ many pairwise disjoint sets $S_{\alpha}$, $\alpha<\omega_{1}$, each having exactly one common element with every $G^{\prime}$-orbit of an element of $B$. Since $m$ is $\sigma$-finite, there exists $\alpha<\omega_{1}$ such that $m\left(S_{\alpha}\right)=0$. Notice that $G^{\prime} B=\bigcup_{g \in G} g S_{\alpha}$ so, again by $\omega_{2}$-additivity of $m, m\left(G^{\prime} B\right)=0$. Thus

$$
m(B)=0
$$

since $B \subseteq G^{\prime} B$. It follows from (1) and (2) that $m(X)=0$ and this contradiction completes the proof of the Claim.

Now fix a subgroup $H$ with the properties stated in the Claim and consider the set $P=\{\langle x, g\rangle \in X \times G: g x \in H x\}$. For every $x \in X$ and $g \in G$ let 
$P_{x}=\{g \in G:\langle x, g\rangle \in P\}$, and $P^{g}=\{x \in X:\langle x, g\rangle \in P\}$. Notice that $P_{x}=\bigcup_{h \in H} h \cdot G_{x}$. Since $G x$ is uncountable, so is the index of the subgroup $G_{x}$ in the group $G$. Hence by $\sigma$-finiteness of the measure $\lambda, \lambda\left(G_{x}\right)=0$. Consequently,

$$
\lambda\left(P_{x}\right)=0 \text { for every } x \in X .
$$

On the other hand, $P^{g}=\{x \in X: g x \in H x\}$. Hence by the property of $H$ expressed in the Claim,

$$
m\left(P^{g}\right)>0 \text { for every } g \in G .
$$

Comparing (3) and (4) we see that a contradiction will be reached as soon as we prove that Fubini's theorem can be applied to the set $P$. We shall use a trick due to Kunen to show that $P$ is measurable with respect to the completion of the product measure $\nu: \mathscr{P}(X) \otimes \mathscr{B} \rightarrow[0,+\infty]$ of $m$ and $\lambda$.

So fix an arbitrary $\varepsilon>0$. Partition the set $X$ into countably many pairwise disjoint subsets $A_{n}$ such that for every $n \in \mathbb{N}, m\left(A_{n}\right)<\infty$. By (3) for every $x \in X$, there exists an open set $U_{x} \subseteq G$ such that $P_{x} \subseteq U_{x}$ and if $x \in A_{n}$ then

$$
\lambda\left(U_{x}\right)<\varepsilon /\left(2^{n+1} \cdot m\left(A_{n}\right)\right) .
$$

Let $U=\left\{\langle x, g\rangle \in X \times G: g \in U_{x}\right\}$. Then $U \in \mathscr{P}(X) \otimes \mathscr{B}$. Indeed, let $\left\{V_{n}: n \in \mathbb{N}\right\}$ be a countable open base for the topology of $G$ and for every $n \in \mathbb{N}$, let $Y_{n}=\left\{x \in X: \forall g \in V_{n}\langle x, g\rangle \in U\right\}$. Then $U=\bigcup_{n \in \mathbb{N}} Y_{n} \times V_{n}$. Hence by Fubini's theorem, $\nu(U)<\varepsilon$. It is now clear how to construct a set $A \in \mathscr{P}(X) \otimes \mathscr{B}$ such that $P \subseteq A$ and $\nu(A)=0$. This completes our argument.

In the following corollary we describe two important cases in which the hypotheses of the theorem are clearly satisfied.

2.2. Corollary. In any of the following cases the uncountability of all G-orbits implies that for every $G$-invariant $\sigma$-finite measure on $X$ there exist nonmeasurable sets:

(i) $G$ is a locally compact Polish group, $X$ is a Hausdorff topological space, and $\langle g, x\rangle \rightarrow g x$ is a Borel-measurable action;

(ii) $G$ is the group of all isometries of a compact, metric, metrically homogeneous space.

\section{Generalizations AND REMARKS}

We present now some possible generalizations of our main result to broader classes of group actions.

First we deal with the case when $G$ is a general locally compact, $\sigma$-compact group. Note that such a group is Polish iff it is second countable. Hence the following result directly generalizes Theorem 2.1 .

3.1. Theorem. Suppose that $G$ is a locally compact, $\sigma$-compact group with topological weight $\kappa$ such that no set of cardinality $\kappa$ carries a universal, nonatomic, $\sigma$-finite measure. Let $\lambda: \mathscr{B} \rightarrow[0,+\infty]$ be the completion of a left-invariant Haar measure on $G$. Suppose further that $G$ acts on a set $X$ in 
such $a$ way that the stabilizers of all points of $X$ are $\lambda$-measurable and all $G$ orbits are uncountable. Then for every $G$-invariant $\sigma$-finite measure on $X$ there exist nonmeasurable sets.

For continuous actions one can drop the restrictions imposed on the weight of $G$.

3.2. Theorem. Suppose that $G$ is a locally compact, $\sigma$-compact group, $X$ is a locally compact, $\sigma$-compact topological space, and the action $\langle g, x\rangle \rightarrow g x$ is continuous. If all $G$-orbits are uncountable, then for every $G$-invariant $\sigma$-finite measure on $X$ there exist nonmeasurable sets.

The proofs of the last two results are beyond the scope of this paper and will be given elsewhere (see [6]).

Now we turn to the case when $G$ is a Polish group. Although it remains open whether for an arbitrary $\sigma$-finite $G$-invariant measure $m$ on $X$ the uncountability of all $G$-orbits guarantees a nonmeasurable set, it does so whenever $m$ is finite (i.e., $m(X)<\infty$ ) .

3.3. Theorem. Suppose that $G$ is a Polish group acting on a set $X$ in such $a$ way that the stabilizers of all points of $X$ are closed in $G$ and all G-orbits are uncountable. Then for every $G$-invariant finite measure on $X$ there exist nonmeasurable sets.

Proof. Suppose, towards a contradiction, that there exists a $G$-invariant, finite, universal measure $m$ on $X$. Let $H$ be a countable dense subgroup of $G$.

Claim. All $H$-orbits are infinite.

For suppose that there is an $x \in X$ such that $|H x|<\omega$. Then there is a finite subset $K$ of $H$ with $H x=\{g x: g \in K\}$. The uncountability of the $G$ orbit of $x$ implies that $G_{x}$ is nowhere dense in $G$. Hence the set $\bigcup_{h \in K} h \cdot G_{x}$ is closed nowhere dense in $G$ and since $H$ is dense, there is $g \in H \backslash \bigcup_{h \in K} h \cdot G_{x}$. But $g x \in K x$, so for some $h \in K, g \in h \cdot G_{x}$, a contradiction.

It follows that $G$ can be partitioned into infinitely many pairwise disjoint sets $S_{n}, n \in \omega$, each having exactly one common element with every $H$-orbit. It is then easy to see that the measure $m$, being universal and $H$-invariant, assigns the same measure to each $S_{n}$ (see [5, Corollary 1.2]). But $m$ is finite and $m(X)=\sum_{n \in \omega} m\left(S_{n}\right)$, so $m(X)=0$, a contradiction.

The case of an infinite measure is covered by the next result, but the uncountability of all $G$-orbits is replaced by an apparently stronger condition.

3.4. Theorem. Suppose that a Polish group $G$ acts on a set $X,|X| \leq 2^{\omega}$, in such a way that the stabilizers of all points of $X$ are Borel in $G$ and the following condition holds:

(*) for every countable subgroup $H$ of $G$ and every $Y \subseteq X$ with $|Y|<2^{\omega}$ there is $g \in G$ such that, for every $y \in Y, g y \notin H y$.

Then for every $G$-invariant $\sigma$-finite measure on $X$ there exist nonmeasurable sets.

Proof (sketch). Suppose, towards a contradiction, that $m$ is a $G$-invariant $\sigma$ finite universal measure on $X$. With no loss of generality assume that there is a set $Z \subseteq X$ such that $m(X \backslash Z)=0$ and every subset of $X$ of cardinality less than $\kappa=|Z|$ has measure $m$ zero. 
Now follow the proof of Theorern 2.1. So let $H$ be a countable subgroup $H$ of $G$ such that, for every $g \in G, m(\{x \in X: g x \in H x\})>0$ and let $P=\{\langle x, g\rangle \in X \times G: g x \in H x\}$. A contradiction will be reached similarly as before, as soon as we show the existence of a $\sigma$-finite outer-regular measure $\lambda$ defined on Borel subsets of $G$ and such that $\lambda\left(P_{x}\right)=0$ for every $x \in Z$.

Note that since $\kappa \leq 2^{\omega},(*)$ tells us that $G \backslash \bigcup_{h \in H, y \in Y} h \cdot G_{y} \neq \varnothing$ for every $Y \subseteq Z$ with $|Y|<\kappa$. Using this, construct a set $L \subseteq G$ such that $|L|=\kappa$ and $\left|L \cap P_{x}\right|<\kappa$ for every $x \in Z$.

Let $f$ be a bijection from $L$ to $Z$. Let $m^{\prime}$ be a finite universal measure on $X$ having the same null sets as the measure $m$ and for every Borel $B \subseteq G$ set:

$$
\lambda(B)=m^{\prime}(f[B \cap L]) .
$$

Then $\lambda$ is a measure on $G$ with the desired properties.

It is conceivable that when $G$ is Polish, then condition $(*)$ in the last result can be weakened to that of the uncountability of all $G$-orbits. The next example shows, however, that if $G$ is assumed only to be a nondiscrete topological group, then even the counterpart of Theorem 3.3 cannot be proved in ZFC, unless the existence of finite, universal, nonatomic measures is refuted altogether.

3.5. Example. Let $m$ be a universal, finite, nonatomic measure on an uncountable set $X$ and let $G$ be the group of all permutations of $X$ which move at most countably many points.

Then the topology of pointwise convergence with respect to the discrete topology on $X$ turns $G$ into a nondiscrete topological group such that, for every $x \in X, G_{x}$ is closed in $G$. Moreover, $X$ is the only $G$-orbit. But $m$ is clearly $G$-invariant.

3.6. Remark. The condition of uncountability of all orbits is essential to avoid the trivial case of the counting measure concentrated on a countable orbit.

3.7. Remark. It is not difficult to prove that under the hypotheses of Theorems 2.1 and 3.1-3.4 the following is true: if $m$ is an arbitrary $G$-invariant $\sigma$-finite measure on $X$, then every set of positive $m$-measure contains a nonmeasurable subset.

Note added in proof. We have recently found an example of an abelian, separable and metric group $G$ of homeomorphisms of $S^{1} \times S^{1}$ such that all $G$-orbits are uncountable, and whenever there exists a finite, universal, nonatomic measure on $\mathbb{R}$, then there is also a $\sigma$-finite, universal, nonatomic measure on $S^{1} \times S^{1}$ which is $G$-invariant.

\section{REFERENCES}

1. F. R. Drake, Set theory: an introduction to large cardinals, North-Holland, Amsterdam, 1974.

2. P. Erdös and R. D. Mauldin, The nonexistence of certain invariant measures, Proc. Amer. Math. Soc. 59 (1976), 321-322.

3. C. Ryll-Nardzewski and R. Telgársky, The nonexistence of universal invariant measures, Proc. Amer. Math. Soc. 69 (1978), 240-242.

4. P. Zakrzewski, The existence of universal invariant measures on large sets, Fund. Math. 133 (1989), 113-124. 
5. $\_$When do equidecomposable sets have equal measures?, Proc. Amer. Math. Soc. 113 (1991), 831-837.

6. _ The uniqueness of Haar measure and set theory (to appear).

Institute of Mathematics, University of Warsaw, ul. Banacha 2, 00-913 Warsaw 59, Poland 